$63^{\text {ème }}$ Congrès de la SFCO, 03005 (2015)

DOI:10.1051/sfco/20156303005

(C) Owned by the authors, published by EDP Sciences, 2015

\title{
Douleurs orofaciales chroniques post-opératoires après chirurgie implantaire : aide au diagnostic à partir d'une revue de la littérature et analyse de cas
}

\author{
Vo Quang S, Descroix V \\ Service d'Odontologie, Groupe Hospitalier Pitié Salpêtrière, Boulevard de l'Hôpital, 75013 Paris
}

L'implantologie orale est une technique considérée comme très sûre et dont le taux de succès au long cours est particulièrement bon. Parmi les complications "précoces » de la chirurgie implantaire (hémorragie, infection), une douleur aiguë doit faire suspecter un traumatisme osseux excessif ou des tissus mous lors de la préparation du site implantaire. La douleur aigue inflammatoire post-opératoire s'amende rapidement en 48 à 72 heures avec l'utilisation d'un traitement antalgique non spécifique. À l'inverse, des douleurs associant des signes d'atteinte nerveuse comme les dysesthésies, les paresthésies ou les anesthésies doivent faire suspecter l'apparition de douleurs post opératoires chroniques.

Les douleurs orofaciales qui persistent à distance de la pose d'un implant dentaire ne sont pas rares même si elles sont considérées comme une complication possible. Ces douleurs chroniques sont aujourd'hui essentiellement considérées comme de nature neuropathique. Ces douleurs peuvent être d'origine iatrogène comme lors de la lésion du nerf alvéolaire inférieur ou encore liées à la désafférentation des fibres nerveuses trijéminales. Elles sont parfois aussi d'origine idiopathique.

Une étude à partir des données récentes de la littérature sera faite ici sur l'épidémiologie, la physiopathologie, les critères diagnostiques, le traitement et la prévention de telles douleurs.

Puis un cas complexe de douleur orofaciale neuropathique d'origine indéterminée chez une femme de 54 ans sera rapporté. Elle se présente à la consultation douleur avec des sensations de brûlure de la langue et d'engourdissement de la commissure labiale droite évoluant depuis plus d'un an et demi et faisant suite à la pose de 2 implants en secteur I. Ses douleurs ne sont pas soulagées par les antalgiques usuels. Un traitement de douleur neuropathique est instauré et la patiente est revue tout les trois mois.

Nom et adresse du conférencier

\section{Soléne VO QUANG}

Service d'Odontologie, Groupe Hospitalier Pitié Salpêtrière

Boulevard de l'Hôpital

75013 Paris (France)

solene.voquang@gmail.com

This is an Open Access article distributed under the terms of the Creative Commons Attribution License 4.0, which permits unrestricted use, distribution, and reproduction in any medium, provided the original work is properly cited. 